Journal of Mathematics and Statistics 6 (1): 10-16, 2010

ISSN 1549-3644

(C) 2010 Science Publications

\title{
Asymptotic Properties of Spectral Estimates of Second-Order with Missed Observations
}

\author{
${ }^{1}$ G.S. Mokaddis, ${ }^{2}$ M.A. Ghazal and ${ }^{3}$ A.E. El-Desokey \\ ${ }^{1}$ Department of Mathematics, Faculty of Science, Ain Shams University, Cairo, Egypt \\ ${ }^{2}$ Department of Mathematics, Faculty of Science, (Damietta) University of Mansoura, Egypt \\ ${ }^{3}$ Department of Mathematics, Faculty of Computer Science, Cairo Academy, Cairo, Egypt
}

\begin{abstract}
Problem statement: As a complement of the periodogram study the asymptotic properties of the spectral density using data window for stationary stochastic process are investigated. Some statistical properties of covariance estimation function with missing observations are studied. Approach: The asymptotic normality was discussed. A numerical example was discussed by using computer programming. Results: The study of time series with missed observations and with the modified periodogram had the same results of the study of the classic time series. Conclusion: Modified periodogram with expanded finite Fourier transformation for time series with missed observation has improved the results of the classic time series.
\end{abstract}

Key words: Continuous time series, modified periodogram, spectral density, spectral measure, autocovariance, data window and asymptotic normality

\section{INTRODUCTION}

Our method of proceeding is to derive the asymptotic moments of: the matrix of sample measures $\mathrm{F}_{\mathrm{XX}}^{(\mathrm{T})}(\lambda)$, the matrix of sample covariances $\mathrm{C}_{\mathrm{XX}}^{(\mathrm{T})}(\mathrm{u})$ and the matrix of sample spectral densities $\mathrm{f}_{\mathrm{XX}}^{(\mathrm{T})}(\lambda)$, including necessary uniform error terms. Our work is mainly based on the properties of the data windows and the matrix of second order smoothing modified periodogram, Ghazal (2005). The properties of the smoothing periodogram using a weight function or data window were discussed in Brillinger (1969); Dahlhaus, 1985. We see that the idea of smoothing the periodograms using data window is an important tool in the spectral analysis of time series. Our purpose is to compare the classic results in the spectral analysis of $r$ - vector valued strictly stationary time series, where all observations are available and the case where some of the observations are randomly missed, using data window.

Related works: Dahlhaus (1985) considered the estimation of spectral measure of stationary process. He discussed the case where $\mathrm{X}(\mathrm{t}), \mathrm{t}$ is a strictly stationary Gaussian time series with real valued components and mean zero. He defined the periodograms, as in Brillinger (1969); Ghazal (2001; 2005) and Ghazal and Farag (2000).

Let $\mathrm{X}(\mathrm{t}) ;(\mathrm{t}=0,1, \ldots, \mathrm{T}-1)$ are be some randomly missing observations. We construct the statistics
$\mathrm{I}_{\mathrm{YY}}^{(\mathrm{T})}(\lambda)(-\infty<\lambda<\infty)$, the matrix of second order smoothing modified periodograms, $\mathrm{F}_{\mathrm{Xx}}^{(\mathrm{T})}(\lambda)$, which is the matrix of second order spectral measures, $\mathrm{f}_{\mathrm{xx}}^{(\mathrm{T})}(\lambda)$ the $r \times r$ matrix of second order spectral densities and $\mathrm{C}_{\mathrm{XX}}^{(\mathrm{T})}(\mathrm{u})(\mathrm{u}=0, \pm 1, \ldots)$ are the matrix of sample covariance.

Suppose that:

$\mathrm{C}_{\mathrm{XX}}(\mathrm{u})=\mathrm{E}\{\mathrm{X}(\mathrm{t}+\mathrm{u}) \overline{\mathrm{X}}(\mathrm{t})\}$

and:

$\sum_{u=-\infty}^{\infty}\left|C_{x x}(u)\right|<\infty$

where, $\left|\mathrm{C}_{\mathrm{xx}}(\mathrm{u})\right|$ denotes the matrix of absolute values, the bar denotes the complex conjugate. We defined $f_{x x}(\lambda)$ the $r \times r$ matrix of second order spectral densities by:

$\mathrm{f}_{\mathrm{xx}}(\lambda)=(2 \pi)^{-1} \sum_{\mathrm{u}=-\infty}^{\infty} \mathrm{c}_{\mathrm{xx}}(\mathrm{u}) \exp (-\mathrm{i} \lambda \mathrm{u})(-\infty<\lambda<\infty)$

and $F_{x x}(\lambda)$ the matrix of second order spectral measures by (Brillinger, 1969):

$\mathrm{F}_{\mathrm{XX}}(\lambda)=\int_{0}^{\lambda} \mathrm{f}_{\mathrm{XX}}(\alpha) \mathrm{d} \alpha(0<\lambda<\pi)$ 
We construct estimates $\mathrm{C}_{\mathrm{XX}}^{(\mathrm{T})}(\mathrm{u}), \mathrm{f}_{\mathrm{XX}}^{(\mathrm{T})}(\lambda)$ and $F_{x x}^{(T)}(\lambda)$ of $C_{x x}(u), f_{x x}(\lambda)$ and $F_{x x}(\lambda)$. These estimates based on $I_{\mathrm{YY}}^{(\mathrm{T})}(\lambda)$, the matrix of second order smoothing modified periodograms. This last is derived from the finite Fourier transform of an observed stretch of data, $\mathrm{X}(\mathrm{t})(\mathrm{t}=0,1, \ldots, \mathrm{T}-1)$ that have some randomly missing observations (Ghazal, 2005). We determine the asymptotic expressions for the cumulates of $\mathrm{F}_{\mathrm{XX}}^{(\mathrm{T})}(\lambda)$, $\mathrm{C}_{\mathrm{XX}}^{(\mathrm{T})}(\mathrm{u})$ and $\mathrm{f}_{\mathrm{XX}}^{(\mathrm{T})}(\lambda)$. Let the r-vector valued time series $X(t)$ have real components $X_{a}(t), \quad(a=\overline{1, r})$. All moments are assumed to exist and we set:

$$
\begin{aligned}
& \mathrm{C}_{\mathrm{a}_{1} \ldots \mathrm{a}_{\mathrm{k}}}\left(\mathrm{t}_{1}, \ldots, \mathrm{t}_{\mathrm{k}-1}\right)=\operatorname{Cum}\left\{\mathrm{x}_{\mathrm{a}_{1}}\left(\mathrm{t}_{1}+\tau\right), \ldots, \mathrm{x}_{\mathrm{a}_{\mathrm{k}-1}}\left(\mathrm{t}_{\mathrm{k}-1}+\tau\right), \mathrm{x}_{\mathrm{a}_{\mathrm{k}}}(\tau)\right\} \\
& \left(\mathrm{a}_{\mathrm{i}}=1, \mathrm{r}, \mathrm{t}=0, \pm 1, \ldots, \mathrm{k}=2,3, \ldots, \tau=0, \pm 1, \ldots\right)
\end{aligned}
$$

Assumption I: $\mathrm{X}(\mathrm{t})$ is a strictly stationary continuous time series and all its moments exist. For each $\mathrm{j}=1, \ldots, \mathrm{k}-1$ and $\mathrm{k}$ - tuple $\mathrm{a}_{1}, \mathrm{a}_{2}, \ldots \mathrm{a}_{\mathrm{k}}$, we have:

$\int_{R^{K-1}}\left|t_{j}\right|\left|C_{a_{1}, \ldots, a_{k-1}}\left(t_{1}, \ldots . t_{k-1}\right) d t_{1}, \ldots, d t_{k-1}\right|<\infty$,

$\mathrm{k}=2,3, .$.

because cumulants are measures of the joint dependence of random variables, (2) is seen to be a form of mixing or asymptotic independence requirement for values of $\mathrm{X}(\mathrm{t})$ well separated in time. If $\mathrm{X}(\mathrm{t})$ satisfies assumption I we may define its cumulant spectral densities by:

$$
\begin{aligned}
& \mathrm{f}_{\mathrm{a}_{1}, \ldots, \mathrm{a}_{\mathrm{k}}}\left(\lambda_{1}, \ldots, \lambda_{\mathrm{k}-1}\right)=(2 \pi)^{-\mathrm{k}+1} \times \\
& \int_{\mathrm{R}^{k-1}}^{\mathrm{T}} \mathrm{C}_{\mathrm{a}_{1}, \ldots, \mathrm{a}_{\mathrm{k}}}\left(\mathrm{t}_{1}, \ldots, \mathrm{t}_{\mathrm{k}-1}\right) \times \exp \left(-\mathrm{i} \sum_{\mathrm{j}=1}^{\mathrm{k}-1} \lambda_{\mathrm{j}} \mathrm{t}_{\mathrm{j}}\right) \mathrm{dt}_{1} \ldots \mathrm{dt}_{\mathrm{k}-1}, \\
& \lambda \in \mathrm{R}, \mathrm{a}_{\mathrm{j}}, 1, \mathrm{rk}=2,3, \ldots
\end{aligned}
$$

Assumption II: Let $d_{a}^{(T)}(t)=d_{a}\left(\frac{t}{T}\right), a=1, r$ be $a$ function which is bounded and has bounded variations and equal zero outside the interval $[0, \mathrm{~T}]$. $\int_{-\pi}^{\pi} d(a) d \alpha=1$ Given $B_{r}>0$ we then set that:

$$
\mathrm{d}^{(\mathrm{T})}(\alpha)=\mathrm{B}_{\mathrm{T}}^{-1} \mathrm{~d}\left(\mathrm{~B}_{\mathrm{T}}^{-1} \alpha\right)
$$

Assumption III: Let U(T) is bounded variation and vanishes for $\mathrm{t}<0, \mathrm{t}>\mathrm{T}-1$. The function $\mathrm{d}_{\mathrm{a}}^{(\mathrm{T})}(\mathrm{t})$ is called a data window and satisfies:
$\frac{1}{\mathrm{~T}} \int_{0}^{\mathrm{T}} \mathrm{d}_{\mathrm{a}}^{(\mathrm{T})} \mathrm{dt} \underset{\mathrm{T} \rightarrow \infty}{\longrightarrow} \int_{0}^{1} \mathrm{~d}_{\mathrm{a}}(\mathrm{u}) \mathrm{du}, \mathrm{a}=\overline{1, \mathrm{r}}$

$\mathrm{U}_{\mathrm{a}_{1} \ldots \mathrm{a}_{k}}^{(\mathrm{T})}\left(\sum_{\mathrm{J}=1}^{\mathrm{k}} \lambda_{\mathrm{J}}\right)=\int_{0}^{\mathrm{T}}\left[\prod_{j=1}^{\mathrm{k}} \mathrm{d}_{\mathrm{a}}^{(\mathrm{T})}(\mathrm{t})\right] \exp \left\{-\mathrm{it} \lambda_{\mathrm{J}}\right\} \mathrm{dt}$

Let $B_{a}(t)(t=0, \pm 1, \ldots)$ be a process independent of $\mathrm{X}(\mathrm{t})$ such that for every t: $\mathrm{P}\{\mathrm{Ba}(\mathrm{t})=1\}=\mathrm{P}_{\mathrm{a}}>0$ $\operatorname{Cum}\left\{B_{a_{1}}\left(t_{1}\right), \ldots, B_{a_{k}}\left(t_{k}\right)\right\}=p_{a_{1} \ldots a_{k}}, a=\overline{1, r}$.

The success of recording an observation not depends on the fail of another and so it is independent. We may then define the modified series:

$\mathrm{Y}(\mathrm{t})=\mathrm{B}(\mathrm{t}) \mathrm{X}(\mathrm{t})$

Where:

$$
B_{a}(t)=\left\{\begin{array}{lc}
1 & \text { if } x(t) \text { is observed } \\
0 & \text { otherwise }
\end{array}\right.
$$

Then $\mathrm{Y}(\mathrm{t})$ is a strictly stationary $\mathrm{r}$ - vector valued time series all of whose moments exist.

In this study $\mathrm{Nr}\left(\mu_{z}, \sum_{z z}\right)$ denote the multivariate normal distribution with mean $\mu_{z}$ and covariance matrix $\sum_{z z}$ where $\mathrm{Z}$ is an $\mathrm{r}$-vector valued random variable having real- valued components.

Let $\mathrm{N}_{\mathrm{r}}^{\mathrm{c}}\left(\mu_{\mathrm{z}}, \sum_{\mathrm{zz}}\right)$, be the complex multivariate normal distribution with mean $\mu_{z}$ and covariance matrix $\sum_{z z}$ where $\mathrm{Z}$ is of complex- valued components.

Structure of the paper would be as following, construct the moments statistics of $\mathrm{F}_{\mathrm{xx}}^{(\mathrm{T})}(\lambda), \mathrm{C}_{\mathrm{xx}}^{(\mathrm{T})}(\mathrm{u})$ and $\mathrm{f}_{\mathrm{XX}}^{(\mathrm{T})}(\lambda)$ by deriving their mean, covariance and cumulate. We construct the expanded finite Fourier transform with data window with missed observations as:

$$
\mathrm{h}_{\mathrm{a}}^{(\mathrm{T})}(\lambda)=\frac{1}{\sqrt{2 \pi \int_{0}^{\mathrm{T}}\left[\mathrm{d}_{\mathrm{a}}^{(\mathrm{T})}(\mathrm{t})\right]^{2}}} \int_{0}^{\mathrm{T}} \mathrm{d}_{\mathrm{a}}^{(\mathrm{T})}(\mathrm{t}) \mathrm{Y}_{\mathrm{a}}(\mathrm{t}) \times
$$

$\exp (-\mathrm{i} \lambda \mathrm{t}) \mathrm{dt}$

where, $\quad \lambda=\mathrm{Rd}_{\mathrm{a}}^{(\mathrm{T})}(\mathrm{t})=\mathrm{d}_{\mathrm{a}}\left(\frac{\mathrm{t}}{\mathrm{T}}\right) \quad$ then the expanded smoothing modified periodogram is defined by:

$$
\mathrm{I}_{\mathrm{ab}}^{(\mathrm{T})}(\lambda)=\left[2 \pi \mathrm{p}_{\mathrm{ab}} \int_{0}^{\mathrm{T}}\left(\mathrm{d}_{\mathrm{a}}^{(\mathrm{T})}(\mathrm{t})\right)^{2}\right]^{-1} \partial_{\mathrm{a}}^{(\mathrm{T})}(\lambda) \overline{\partial_{\mathrm{b}}^{(\mathrm{T})}(\lambda)}
$$


The bar denotes the complex conjugate and:

$\partial_{a}^{(T)}(\lambda)=\int_{0}^{T} d_{a}^{(T)}(t) Y_{a}(t) \exp (-i \lambda t) d t$

$\lambda \in \mathrm{R} ; \mathrm{a}, \mathrm{b}=\overline{1, \mathrm{r}}$ and $\mathrm{d}_{\mathrm{a}}^{(\mathrm{T})}(\mathrm{t})$ is a data window function equal zero outside the interval $[0, T]$.

Asymptotic moments of spectral measure; autocovariance and spectral density function:

Theorem 1: Let $\mathrm{Ya}(\mathrm{t})=\mathrm{Xa}(\mathrm{t}) \mathrm{Ha}(\mathrm{t}),(\mathrm{a}=\overline{1, \mathrm{r}})$ are missed observations on a strictly stationary continuous time series $\mathrm{Xa}(\mathrm{t}),(\mathrm{a}=\overline{1, \mathrm{r}}),(\mathrm{t}=0, \pm 1, \ldots)$ which satisfies Assumption I with mean zero and let $\mathrm{F}_{\mathrm{XX}}^{(\mathrm{T})}(\lambda)$ is defined then:

$\mathrm{E}\left\{\mathrm{F}_{\mathrm{ab}}^{(\mathrm{T})}(\lambda)\right\}=\mathrm{P}_{\mathrm{ab}} \mathrm{F}_{\mathrm{ab}}(\lambda)+\mathrm{O}\left(\mathrm{T}^{-1}\right)$

with $\mathrm{O}\left(\mathrm{T}^{-1}\right)$ uniform in $\lambda$.

$\operatorname{Cov}\left\{\mathrm{F}_{\mathrm{a}_{1} \mathrm{~b}_{1}}^{(\mathrm{T})}\left(\lambda_{1}\right) \mathrm{F}_{\mathrm{a}_{2} \mathrm{~b}_{2}}^{(\mathrm{T})}\left(\lambda_{2}\right)\right\}=(2 \pi) \mathrm{P}_{\mathrm{a}_{1} \mathrm{a}_{2} \mathrm{~b}_{1} \mathrm{~b}_{2}} \times$

$\left[\mathrm{U}_{\mathrm{a}_{1} \mathrm{~b}_{1}}^{(\mathrm{T})}(0) \mathrm{U}_{\mathrm{a}_{2} \mathrm{~b}_{2}}^{(\mathrm{T})}(0)\right]^{-1}\left[\mathrm{U}_{\mathrm{a}_{1} \mathrm{a}_{2} \mathrm{~b}_{1} \mathrm{~b}_{2}}^{(\mathrm{T})}(0)\right] \times$

$\left[\int_{-\infty}^{\lambda_{1}} f_{a_{1} a_{2}}\left(\alpha_{1}\right) f_{b_{1} b_{2}}\left(-\alpha_{1}\right) d \alpha_{1}+\int_{-\infty}^{\lambda_{1}} f_{a_{1} b_{2}}\left(\alpha_{1}\right) f_{b_{1} a_{2}}\left(-\alpha_{1}\right) d \alpha_{1}\right]$

$+\mathrm{O}\left(\mathrm{T}^{-1}\right)$

and

$$
\operatorname{Cum}\left\{\mathrm{F}_{\mathrm{a}_{1} \mathrm{~b}_{1}}^{(\mathrm{T})}\left(\lambda_{1}\right), \ldots, \mathrm{F}_{\mathrm{a}_{\mathrm{k}} \mathrm{b}_{\mathrm{k}}}^{(\mathrm{T})}\left(\lambda_{\mathrm{k}}\right)\right\}=\prod_{\mathrm{j}=1}^{\mathrm{k}} \mathrm{P}_{\mathrm{c}_{\mathrm{j}} \mathrm{d}_{\mathrm{j}}} \mathrm{O}\left(\mathrm{T}^{-\mathrm{k}+1}\right), \mathrm{k}=2,3, \ldots(10
$$

where the error terms are uniform in each case.

Lemma 1: Let $\mathrm{Ya}(\mathrm{t})=\mathrm{Xa}(\mathrm{t}) \mathrm{Ha}(\mathrm{t}),(\mathrm{a}=\overline{1, \mathrm{r}})$ are missed observations on the strictly stationary continuous series $\mathrm{Xa}(\mathrm{t}),(\mathrm{a}=\overline{1, \mathrm{r}}),(\mathrm{t}=0, \pm 1, \ldots)$ which satisfies assumption I with mean zero under the condition of Theorem 1 we have:

$$
\begin{aligned}
& \mathrm{E}\left\{\mathrm{F}_{\mathrm{ab}}^{(\mathrm{T})}(\lambda)\right\} \underset{\mathrm{T} \rightarrow \infty}{\longrightarrow} \mathrm{P}_{\mathrm{ab}} \mathrm{F}_{\mathrm{ab}}(\lambda), \\
& \operatorname{Cov}\left\{\mathrm{F}_{\mathrm{a}_{1} \mathrm{~b}_{1}}^{(\mathrm{T})}\left(\lambda_{1}\right), \mathrm{F}_{\mathrm{a}_{2} \mathrm{~b}_{2}}^{(\mathrm{T})}\left(\lambda_{2}\right)\right\} \underset{\mathrm{T} \rightarrow \infty}{\longrightarrow} 0, \\
& \operatorname{Cum}\left\{\mathrm{F}_{\mathrm{a}_{1} \mathrm{~b}_{1}}^{(\mathrm{T})}\left(\lambda_{1}\right), \ldots, \mathrm{F}_{\mathrm{a}_{k} \mathrm{~b}_{k}}^{(\mathrm{T})}\left(\lambda_{k}\right)\right\} \underset{\mathrm{T} \rightarrow \infty}{\longrightarrow} 0
\end{aligned}
$$

For all $\mathrm{a}_{\mathrm{j}}, \mathrm{b}_{\mathrm{j}}=\overline{1, \mathrm{r}}=0 \leq \lambda<\infty, \mathrm{j}=\overline{1, \ldots, \mathrm{k}} \cdot \mathrm{k}=1,2 \ldots$, as an estimate of $\operatorname{CXX~(u)~we~consider:~}$

$$
\mathrm{m}_{\mathrm{XX}}^{(\mathrm{T})}(\mathrm{u})=\int_{-\infty}^{\infty} \mathrm{I}_{\mathrm{XX}}^{(\mathrm{T})}(\alpha) \exp (\mathrm{iu} \alpha) \mathrm{d} \alpha
$$

we have the following theorem.

Theorem 2: Let $\mathrm{Ya}(\mathrm{t})=\mathrm{Xa}(\mathrm{t}) \mathrm{Ha}(\mathrm{t}),(\mathrm{a}=\overline{1, \mathrm{r}}) \quad$ are missed observations on a strictly stationary continuous time series $\mathrm{Xa}(\mathrm{t}),(\mathrm{a}=\overline{1, \mathrm{r}}),(\mathrm{t}=0, \pm 1, \ldots)$ which satisfies assumption I with mean zero then:

$\mathrm{E}\left\{\mathrm{m}_{\mathrm{ab}}^{(\mathrm{T})}(\mathrm{u})\right\}=\mathrm{P}_{\mathrm{ab}} \mathrm{m}_{\mathrm{ab}}(\mathrm{u})+\mathrm{O}\left(\mathrm{T}^{-1}\right)$

$\operatorname{Cov}\left\{\mathrm{m}_{\mathrm{a}_{1} \mathrm{~b}_{1}}^{(\mathrm{T})}\left(\mathrm{u}_{1}\right), \mathrm{m}_{\mathrm{a}_{2} \mathrm{~b}_{2}}^{(\mathrm{T})}\left(\mathrm{u}_{2}\right)\right\}=2 \pi\left(\mathrm{U}_{\mathrm{a}_{2} \mathrm{~b}_{2}}^{(\mathrm{T})}(0) \mathrm{U}_{\mathrm{a}_{1} \mathrm{~b}_{1}}^{(\mathrm{T})}(0)\right)^{-1} \times$

$\mathrm{U}_{\mathrm{a}_{1} \mathrm{a}_{2} \mathrm{~b}_{2} \mathrm{~b}_{2}}^{(t)}(0) \times\left\{\begin{array}{l}\mathrm{P}_{\mathrm{a}_{1} \mathrm{a}_{2}} \mathrm{P}_{\mathrm{b}_{1} \mathrm{~b}_{2}} \int_{-\infty}^{\infty} \exp \left\{\mathrm{i} \alpha\left(\mathrm{u}_{1}-\mathrm{u}_{2}\right)\right\} \mathrm{f}_{\mathrm{a}_{1} \mathrm{a}_{2}}(\alpha) \mathrm{f}_{\mathrm{b}_{1} \mathrm{~b}_{2}} \\ \left(-\alpha_{1}\right) \mathrm{d} \alpha\end{array}\right.$

$\left.+\mathrm{P}_{\mathrm{a}_{1} \mathrm{~b}_{2}} \mathrm{P}_{\mathrm{b}_{1} \mathrm{a}_{2}} \int_{-\infty}^{\infty} \exp \left\{i \alpha\left(\mathrm{u}_{1}+\mathrm{u}_{2}\right)\right\} \mathrm{f}_{\mathrm{a}_{1} \mathrm{~b}_{2}}(\alpha) \mathrm{f}_{\mathrm{b}_{1} \mathrm{a}_{2}}(-\alpha) \mathrm{d} \alpha\right\}$

$+\mathrm{O}\left(\mathrm{T}^{-1}\right)$

and:

$\operatorname{Cum}\left\{\mathrm{m}_{\mathrm{a}_{1} \mathrm{~b}_{1}}^{(\mathrm{T})}\left(\mathrm{u}_{1}\right), \ldots, \mathrm{m}_{\mathrm{a}_{\mathrm{k}} \mathrm{b}_{\mathrm{k}}}^{(\mathrm{T})}\left(\mathrm{u}_{\mathrm{k}}\right)\right\}=\prod_{\mathrm{j}=1}^{\mathrm{k}} \mathrm{P}_{\mathrm{c}_{\mathrm{j}} \mathrm{d}_{j}} \mathrm{O}\left(\mathrm{T}^{-\mathrm{k}+1}\right)$, $\mathrm{k}=2,3, \ldots$

for all $a_{j}, b_{j}=\overline{1, r}, u_{j}=0, \pm 1, \ldots, j=\overline{1, k}$ The error terms are uniform in each case.

From the previous theorem we have the following lemma:

Lemma 2: Let $\mathrm{Ya}(\mathrm{t})=\mathrm{Xa}(\mathrm{t}) \mathrm{Ha}(\mathrm{t}),(\mathrm{a}=\overline{1, \mathrm{r}})$ are missed observations on a strictly stationary continuous time series $\mathrm{Xa}(\mathrm{t}), \quad(\quad \mathrm{a}=\overline{1, \mathrm{r}})), \quad(\mathrm{t}=0, \pm 1, \ldots) \quad$ which satisfies assumption I with mean zero then we have:

$$
\begin{aligned}
& \mathrm{E}\left\{\mathrm{m}_{\mathrm{ab}}^{(\mathrm{T})}(\mathrm{u})\right\} \underset{\mathrm{T} \rightarrow \infty}{\longrightarrow} \mathrm{P}_{\mathrm{a}} \mathrm{P}_{\mathrm{b}} \mathrm{m}_{\mathrm{ab}}(\mathrm{u}) \\
& \operatorname{Cov}\left\{\mathrm{m}_{\mathrm{a}_{1} \mathrm{~b}_{1}}^{(\mathrm{T})}\left(\mathrm{u}_{1}\right), \mathrm{m}_{\mathrm{a}_{2} \mathrm{~b}_{2}}^{(\mathrm{T})}\left(\mathrm{u}_{2}\right)\right\} \underset{\mathrm{T} \rightarrow \infty}{\longrightarrow} 0 \\
& \operatorname{Cum}\left\{\mathrm{m}_{\mathrm{a}_{1} \mathrm{~b}_{1}}^{(\mathrm{T})}\left(\mathrm{u}_{1}\right), \ldots, \mathrm{m}_{\mathrm{a}_{\mathrm{k}} \mathrm{b}_{\mathrm{k}}}^{(\mathrm{T})}\left(\mathrm{u}_{\mathrm{k}}\right)\right\} \underset{\mathrm{T} \rightarrow \infty}{\longrightarrow} 0
\end{aligned}
$$

for all $a_{j}, b_{j}=\overline{1, r}, j=\overline{1, k}$.

Let $\mathrm{f}_{\mathrm{XX}}^{(\mathrm{T})}(\mathrm{u})$ be defined as:

$\mathrm{f}_{\mathrm{XX}}^{(\mathrm{T})}(\lambda)=\int_{-\infty}^{\infty} \Psi^{(\mathrm{T})}(\lambda-\alpha) \mathbf{I}_{\mathrm{xX}}^{(\mathrm{T})}(\alpha) \mathrm{d} \alpha$ 
Assumption IV: Let $\Psi(\alpha), \alpha \in \mathrm{R}$ is a weight function which is bounded and has bonded first derivative such that:

$$
\int_{-\infty}^{\infty} \Psi(\alpha) \mathrm{d} \alpha=1
$$

Given BT>0, we then set:

$$
\Psi^{(\mathrm{T})}(\alpha)=\mathrm{B}_{\mathrm{T}}^{-1} \Psi\left(\mathrm{B}_{\mathrm{T}}^{-1} \alpha\right)
$$

Theorem 3: Let $\mathrm{Ya}(\mathrm{t})=\mathrm{Xa}(\mathrm{t}) \mathrm{Ha}(\mathrm{t}), \quad(\mathrm{a}=\overline{1, \mathrm{r}})$ are missed observations on the strictly stationary Continuous series $\mathrm{Xa}(\mathrm{t}), \quad(\mathrm{a}=\overline{1, \mathrm{r}}), \quad(\mathrm{t}=0, \pm 1, \ldots)$ which satisfies assumption I with mean zero, then:

$$
\begin{aligned}
& \mathrm{E}\left\{\mathrm{f}_{\mathrm{ab}}^{(\mathrm{T})}(\lambda)\right\}=\mathrm{P}_{\mathrm{ab}} \int_{-\infty}^{\infty} \Psi_{\mathrm{ab}}(\alpha) \mathrm{f}_{\mathrm{ab}}\left(\lambda-\alpha \mathrm{B}_{\mathrm{T}}\right) \mathrm{d} \alpha+\mathrm{O}\left(\mathrm{T}^{-1}\right) \\
& \operatorname{Cov}\left\{\mathrm{f}_{\mathrm{a}_{1} \mathrm{~b}_{1}}^{(\mathrm{T})}\left(\lambda_{1}\right), \mathrm{f}_{\mathrm{a}_{2} \mathrm{~b}_{2}}^{(\mathrm{T})}\left(\lambda_{2}\right)\right\}=2 \pi \mathrm{U}_{\mathrm{a}_{1} \mathrm{a}_{2} \mathrm{~b}_{1} \mathrm{~b}_{2}}^{(\mathrm{T})}(0) \times \\
& \left(\mathrm{U}_{\mathrm{a}_{1} \mathrm{~b}_{1}}^{(\mathrm{T})}(0) \mathrm{U}_{\mathrm{a}_{2} \mathrm{~b}_{2}}^{(\mathrm{T})}(0)\right)^{-1} \times \\
& \left\{\mathrm{P}_{\mathrm{a}_{1} \mathrm{a}_{2}} \mathrm{P}_{\mathrm{b}_{1} \mathrm{~b}_{2}} \int_{-\infty}^{\infty} \Psi_{\mathrm{a}_{1} \mathrm{~b}_{1}}(\alpha) \Psi_{\mathrm{a}_{2} \mathrm{~b}_{2}}\left(\lambda_{2}-\lambda_{1}+\alpha\right) \mathrm{f}_{\mathrm{a}_{1} \mathrm{a}_{2}}\left(\lambda_{1}-\alpha\right) \times\right. \\
& \mathrm{f}_{\mathrm{b}_{1} \mathrm{~b}_{2}}\left(\alpha-\lambda_{1}\right) \mathrm{d} \alpha+\mathrm{P}_{\mathrm{a}_{1} \mathrm{~b}_{2}} \mathrm{P}_{\mathrm{b}_{1} \mathrm{a}_{2}} \int_{-\infty}^{\infty} \Psi_{\mathrm{a}_{1} \mathrm{~b}_{1}}(\alpha) \Psi_{\mathrm{a}_{2} \mathrm{~b}_{2}}\left(\lambda_{2}+\lambda_{1}-\alpha\right) \times \\
& \left.\mathrm{f}_{\mathrm{a}_{1} \mathrm{~b}_{2}}\left(\lambda_{1}-\alpha\right) \mathrm{f}_{\mathrm{b}_{1} \mathrm{a}_{2}}\left(\alpha-\lambda_{1}\right) \mathrm{d} \alpha\right\}+\mathrm{O}\left(\mathrm{T}^{-2}\right) \text {, when } \mathrm{B}_{\mathrm{T}}=1 \\
& \operatorname{Cov}\left\{\mathrm{f}_{\mathrm{a}_{1} \mathrm{~b}_{1}}^{(\mathrm{T})}\left(\lambda_{1}\right), \mathrm{f}_{\mathrm{a}_{2} \mathrm{~b}_{2}}^{(\mathrm{T})}\left(\lambda_{2}\right)\right\}=2 \pi \mathrm{B}_{\mathrm{T}}^{-1} \mathrm{U}_{\mathrm{a}_{1} \mathrm{a}_{2} \mathrm{~b}_{1} \mathrm{~b}_{2}}^{(\mathrm{T})}(0) \times \\
& \left(\mathrm{U}_{\mathrm{a}_{1} \mathrm{~b}_{1}}^{(\mathrm{T})}(0) \mathrm{U}_{\mathrm{a}_{2} \mathrm{~b}_{2}}^{(\mathrm{T})}(0)\right)^{-1}\left\{\int_{-\infty}^{\infty} \Psi_{\mathrm{a}_{1} \mathrm{~b}_{1}}(\alpha) \Psi_{\mathrm{a}_{2} \mathrm{~b}_{2}}(\alpha) \mathrm{d} \alpha\right\} \times \\
& {\left[\delta\left(\lambda_{1}-\lambda_{2}\right) \mathrm{P}_{\mathrm{a}_{1} \mathrm{a}_{2}} \mathrm{P}_{\mathrm{b}_{1} \mathrm{~b}_{2}} \mathrm{f}_{\mathrm{a}_{1} \mathrm{a}_{2}}\left(\lambda_{1}\right) \mathrm{f}_{\mathrm{b}_{1} \mathrm{~b}_{2}}\left(-\lambda_{1}\right)+\right.} \\
& \left.\delta\left(\lambda_{1}+\lambda_{2}\right) \mathrm{P}_{\mathrm{a}_{1} \mathrm{~b}_{2}} \mathrm{P}_{\mathrm{b}_{1} \mathrm{a}_{2}} \mathrm{f}_{\mathrm{a}_{1} \mathrm{~b}_{2}}\left(\lambda_{1}\right) \mathrm{f}_{\mathrm{b}_{1} \mathrm{a}_{2}}\left(-\lambda_{1}\right)\right]+\mathrm{O}\left(\mathrm{B}_{\mathrm{T}}^{-1} \mathrm{~T}^{-2}\right)
\end{aligned}
$$

when $\mathrm{B}_{\mathrm{T}} \rightarrow 0, \mathrm{~B}_{\mathrm{T}} \mathrm{T} \rightarrow \infty$ as $\mathrm{T} \rightarrow \infty$. Also:

$$
\begin{aligned}
& \operatorname{Cum}\left\{\mathrm{f}_{\mathrm{a}_{1} \mathrm{~b}_{1}}^{(\mathrm{T})}\left(\lambda_{1}\right), \ldots, \mathrm{f}_{\mathrm{a}_{\mathrm{k}} \mathrm{b}_{\mathrm{k}}}^{(\mathrm{T})}\left(\lambda_{\mathrm{k}}\right)\right\}= \\
& \begin{cases}\left(\prod_{\mathrm{j}=1}^{\mathrm{k}} \mathrm{P}_{\mathrm{c}_{\mathrm{j}} \mathrm{d}_{j}}\right) \mathrm{O}\left(\mathrm{T}^{-\mathrm{k}+1}\right), & \mathrm{B}_{\mathrm{T}}=1, \mathrm{k} \geq 2, \\
\left(\prod_{j=1}^{\mathrm{k}} \mathrm{P}_{\mathrm{c}_{\mathrm{j}} \mathrm{d}_{\mathrm{j}}}\right) \mathrm{O}\left(\mathrm{B}_{\mathrm{T}}^{-\mathrm{k}+1} \mathrm{~T}^{-\mathrm{k}+1}\right), & \mathrm{B}_{\mathrm{T}} \rightarrow 0, \mathrm{~B}_{\mathrm{T}} \mathrm{T} \rightarrow \infty\end{cases}
\end{aligned}
$$

The following lemma indicates that $f_{a b}^{(T)}(\lambda)$ is an asymptotically unbiased estimate of $\mathrm{f}_{\mathrm{ab}}(\lambda)$, if $\lambda \neq 0$ and $\mathrm{B}_{\mathrm{T}} \rightarrow 0$ as $\mathrm{T} \rightarrow \infty$.

Lemma 3: Under the conditions of Theorem 3, if $\mathrm{B}_{\mathrm{T}} \rightarrow 0, \quad \mathrm{~B}_{\mathrm{T}} \rightarrow \infty$ as $\mathrm{T} \rightarrow \infty$, then:

$$
\begin{aligned}
& \mathrm{E}\left\{\mathrm{f}_{\mathrm{ab}}^{(\mathrm{T})}(\lambda)\right\} \underset{\mathrm{T} \rightarrow \infty}{\longrightarrow} \mathrm{P}_{\mathrm{ab}} \mathrm{f}_{\mathrm{ab}}(\lambda), \\
& \operatorname{Cov}\left\{\mathrm{f}_{\mathrm{a}_{1} \mathrm{~b}_{1}}^{(\mathrm{T})}\left(\lambda_{1}\right), \mathrm{f}_{\mathrm{a}_{2} \mathrm{~b}_{2}}^{(\mathrm{T})}\left(\lambda_{2}\right)\right\} \underset{\mathrm{T} \rightarrow \infty}{\longrightarrow} 0, \\
& \operatorname{Cum}\left\{\mathrm{f}_{\mathrm{a}_{1} \mathrm{~b}_{1}}^{(\mathrm{T})}\left(\lambda_{1}\right), \ldots, \mathrm{f}_{\mathrm{a}_{\mathrm{k}} \mathrm{b}_{\mathrm{k}}}^{(\mathrm{T})}\left(\lambda_{\mathrm{k}}\right)\right\} \underset{\mathrm{T} \rightarrow \infty}{\longrightarrow} 0
\end{aligned}
$$

for all $a_{j}, b_{j}=\overline{1, r}, \lambda \lambda_{j} \lambda \in[-\pi, \pi], j=\overline{1, k}, k=1,2, \ldots$, .

Asymptotic normality: Now, turning to finding the asymptotic distributions of $\mathrm{F}_{\mathrm{XX}}^{(\mathrm{T})}(\lambda), \mathrm{m}_{\mathrm{xx}}^{(\mathrm{T})}(\lambda), \mathrm{f}_{\mathrm{xx}}^{(\mathrm{T})}(\lambda)$ which are based on the asymptotic distribution of $\mathrm{I}_{\mathrm{ab}}^{(\mathrm{T})}(\lambda), \quad \mathrm{a}, \mathrm{b}=\overline{1, \mathrm{r}}, \lambda \in[-\pi, \pi]$. We shall prove that the asymptotic distribution of previous statistics is normal with mean zero the asymptotic normality has been demonstrated, under various conditions, by (Ghazal, 2005; Dahlhaus, 1985; Ghazal and Farag, 2000; Ghazal and Elhassanein, 2006).

Theorem 4: Let $\mathrm{Ya}(\mathrm{t})=\mathrm{Xa}(\mathrm{t}) \mathrm{Ha}(\mathrm{t}),(\mathrm{a}=\overline{1, \mathrm{r}})$ are missed observations on a strictly stationary continuous time series $\mathrm{Xa}(\mathrm{t}),((\mathrm{a}=\overline{1, \mathrm{r}})),(\mathrm{t}=0, \pm 1, \ldots)$ which satisfies assumption I with mean zero. Then:

$$
\mathrm{T}^{\frac{1}{2}}\left\{\mathrm{~F}_{\mathrm{XX}}^{(\mathrm{T})}\left(\lambda_{1}\right)-\mathrm{F}_{\mathrm{XX}}\left(\lambda_{1}\right)\right\}, \ldots, \mathrm{T}^{\frac{1}{2}}\left\{\mathrm{~F}_{\mathrm{XX}}^{(\mathrm{T})}\left(\lambda_{\mathrm{k}}\right)-\mathrm{F}_{\mathrm{XX}}\left(\lambda_{\mathrm{k}}\right)\right\}
$$

are asymptotically jointly multivariate normal with covariance structure:

$$
\begin{aligned}
& \operatorname{Lim}_{\mathrm{T} \rightarrow \infty} \operatorname{Cov}\left[\begin{array}{c}
\mathrm{T}^{\frac{1}{2}}\left\{\mathrm{~F}_{\mathrm{a}_{1} \mathrm{~b}_{1}}^{(\mathrm{T})}\left(\lambda_{1}\right)-\mathrm{F}_{\mathrm{a}_{1} \mathrm{~b}_{1}}\left(\lambda_{1}\right)\right\}, \\
\mathrm{T}^{\frac{1}{2}}\left\{\mathrm{~F}_{\mathrm{a}_{2} \mathrm{~b}_{2}}^{(\mathrm{T})}\left(\lambda_{2}\right)-\mathrm{F}_{\mathrm{a}_{2} \mathrm{~b}_{2}}\left(\lambda_{2}\right)\right\}
\end{array}\right]=(2 \pi)\left[\mathrm{U}_{\mathrm{a}_{1} \mathrm{~b}_{1}}^{(\mathrm{T})}(0) \mathrm{U}_{\mathrm{a}_{2} \mathrm{~b}_{2}}^{(\mathrm{T})}(0)\right]^{-1} \\
& {\left[\mathrm{U}_{\mathrm{a}_{1} \mathrm{a}_{2} \mathrm{~b}_{1} \mathrm{~b}_{2}}^{(\mathrm{T})}(0)\right]\left[\mathrm{P}_{\mathrm{a}_{1} \mathrm{a}_{2}} \mathrm{P}_{\mathrm{b}_{1} \mathrm{~b}_{2}} \int_{-\infty}^{\lambda_{1}} \mathrm{f}_{\mathrm{a}_{1} \mathrm{a}_{2}}\left(\alpha_{1}\right) \times \mathrm{f}_{\mathrm{b}_{1} \mathrm{~b}_{2}}\left(-\alpha_{1}\right) \mathrm{d} \alpha_{1}\right.} \\
& \left.+\mathrm{P}_{\mathrm{a}_{1} \mathrm{~b}_{2}} \mathrm{P}_{\mathrm{a}_{2} \mathrm{~b}_{1}} \int_{-\infty}^{\lambda_{1}} \mathrm{f}_{\mathrm{a}_{1} \mathrm{~b}_{2}}\left(\alpha_{1}\right) \mathrm{f}_{\mathrm{b}_{1} \mathrm{a}_{2}}\left(-\alpha_{1}\right) \mathrm{d} \alpha_{1}\right]
\end{aligned}
$$

for all $\mathrm{a}_{\mathrm{j}}, \mathrm{b}_{\mathrm{j}}=\overline{1, \mathrm{r}}, \lambda_{\mathrm{j}} \in[-\pi, \pi], \mathrm{j}=\overline{1, \mathrm{k}}, \mathrm{k}=1,2, \ldots$

Theorem 5: Let $\mathrm{Ya}(\mathrm{t})=\mathrm{Xa}(\mathrm{t}) \mathrm{Ha}(\mathrm{t}), \quad(\mathrm{a}=\overline{1, \mathrm{r}}) \quad$ are missed observations on a strictly stationary continuous 
time series $\mathrm{Xa}(\mathrm{t}),(\mathrm{a}=\overline{1, \mathrm{r}}),(\mathrm{t}=0, \pm 1, \ldots) \quad$ which satisfies assumption I with mean zero. Then:

$$
\mathrm{T}^{\frac{1}{2}}\left\{\mathrm{~m}_{\mathrm{a}_{1} \mathrm{~b}_{1}}^{(\mathrm{T})}\left(\mathrm{u}_{1}\right)-\mathrm{m}_{\mathrm{a}_{1} \mathrm{~b}_{1}}\left(\mathrm{u}_{1}\right)\right\}, \ldots, \mathrm{T}^{\frac{1}{2}}\left\{\mathrm{~m}_{\mathrm{a}_{\mathrm{k}} \mathrm{b}_{\mathrm{k}}}^{(\mathrm{T})}\left(\mathrm{u}_{\mathrm{k}}\right)-\mathrm{m}_{\mathrm{a}_{\mathrm{k}} \mathrm{b}_{\mathrm{k}}}\left(\mathrm{u}_{\mathrm{k}}\right)\right\}
$$

are asymptotically jointly multivariate normal with covariance structure given by:

$$
\begin{aligned}
& \operatorname{Lim}_{\mathrm{T} \rightarrow \infty} \operatorname{Cov}\left[\begin{array}{c}
\mathrm{T}^{\frac{1}{2}}\left\{\mathrm{~m}_{\mathrm{a}_{1} \mathrm{~b}_{1}}^{(\mathrm{T})}\left(\mathrm{u}_{1}\right)-\mathrm{m}_{\mathrm{a}_{1} \mathrm{~b}_{1}}\left(\mathrm{u}_{1}\right)\right\}, \\
\mathrm{T}^{\frac{1}{2}}\left\{\mathrm{~m}_{\mathrm{a}_{2} \mathrm{~b}_{2}}^{(\mathrm{T})}\left(\mathrm{u}_{2}\right)-\mathrm{m}_{\mathrm{a}_{2} \mathrm{~b}_{2}}\left(\mathrm{u}_{2}\right)\right\}
\end{array}\right] \\
& 2 \pi\left(\mathrm{U}_{\mathrm{a}_{1} \mathrm{~b}_{1}}^{(\mathrm{T})}(0) \mathrm{U}_{\mathrm{a}_{2} \mathrm{~b}_{2}}^{(\mathrm{T})}(0)\right)^{-1} \mathrm{U}_{\mathrm{a}_{1} \mathrm{a}_{2} \mathrm{~b}_{1} \mathrm{~b}_{2}}(0) \times \\
& {\left[\mathrm{P}_{\mathrm{a}_{1} \mathrm{a}_{2}} \mathrm{P}_{\mathrm{b}_{1} \mathrm{~b}_{2}} \int_{-\infty}^{\infty} \exp \left\{i \alpha\left(\mathrm{u}_{1}-\mathrm{u}_{2}\right)\right\} \mathrm{f}_{\mathrm{a}_{1} \mathrm{a}_{2}}\left(\alpha_{1}\right) \mathrm{f}_{\mathrm{b}_{1} \mathrm{~b}_{2}}\left(-\alpha_{1}\right) \mathrm{d} \alpha_{1}+\right.} \\
& \left.\mathrm{P}_{\mathrm{a}_{1} \mathrm{~b}_{2}} \mathrm{P}_{\mathrm{b}_{1} \mathrm{a}_{2}} \int_{-\infty}^{\infty} \exp \left\{i \alpha\left(\mathrm{u}_{1}+\mathrm{u}_{2}\right)\right\} \mathrm{f}_{\mathrm{a}_{1} \mathrm{~b}_{2}}\left(\alpha_{1}\right) \mathrm{f}_{\mathrm{b}_{1} \mathrm{a}_{2}}\left(-\alpha_{1}\right) \mathrm{d} \alpha_{1}\right]
\end{aligned}
$$

for all $\mathrm{a}_{\mathrm{j}}, \mathrm{b}_{\mathrm{j}}=\overline{1, \mathrm{r}}, \lambda_{\mathrm{j}} \in[-\pi, \pi], \mathrm{j}=\overline{1, \mathrm{k}}, \mathrm{k}=1,2, \ldots$ Theorem 4 Let $\mathrm{Ya}(\mathrm{t})=\mathrm{Xa}(\mathrm{t}) \mathrm{Ha}(\mathrm{t}), \quad(\mathrm{a}=\overline{1, \mathrm{r}})$ are missed observations on a strictly stationary continuous time series $\mathrm{Xa}(\mathrm{t}), \quad(\mathrm{a}=\overline{1, \mathrm{r}}), \quad(\mathrm{t}=0, \pm 1, \ldots) \quad$ which satisfies assumption I with mean zero. If $\mathrm{B}_{\mathrm{T}} \mathrm{T} \rightarrow \infty$ as $\mathrm{T} \rightarrow \infty$; then:

$$
\begin{aligned}
& \left(\mathrm{B}_{\mathrm{T}} \mathrm{T}^{\frac{1}{2}}\right)\left\{\mathrm{f}_{\mathrm{XX}}^{(\mathrm{T})}\left(\lambda_{1}\right)-\mathrm{E}\left\{\mathrm{f}_{\mathrm{XX}}^{(\mathrm{T})}\left(\lambda_{1}\right)\right\}\right\}, \ldots, \\
& \left(\mathrm{B}_{\mathrm{T}} \mathrm{T}^{\frac{1}{2}}\right)\left\{\mathrm{f}_{\mathrm{XX}}^{(\mathrm{T})}\left(\lambda_{\mathrm{k}}\right)-\mathrm{E}\left\{\mathrm{f}_{\mathrm{XX}}^{(\mathrm{T})}\left(\lambda_{\mathrm{k}}\right)\right\}\right\}
\end{aligned}
$$

are asymptotically normal with mean zero and covariance structure indicated in Theorem 5.

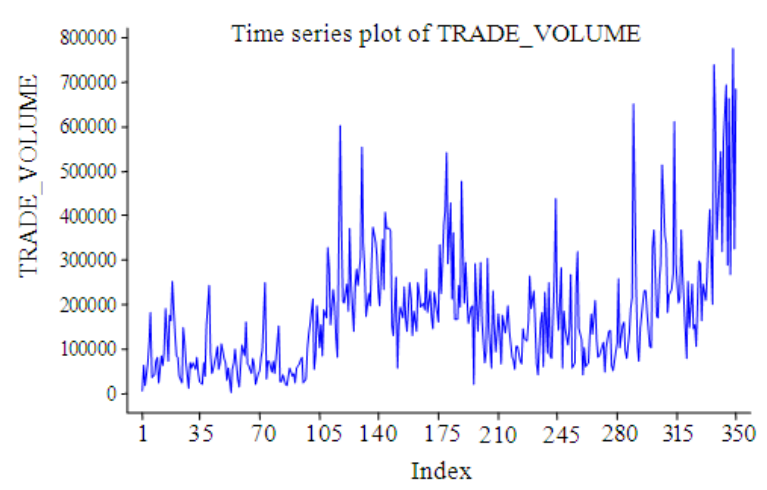

Fig. 1: The weakly trade volume data, $\mathrm{X}(\mathrm{t}), 2003-2008$
Example: In this example, we will comparison between our results, spectral analysis of strictly stationary time series with some missing observations and the classic results, where all observations are available. Data is available at www.egidegypt.com.

Let $\mathrm{Xa}(\mathrm{t}),(\mathrm{t}=0, \pm 1, \ldots)$ be a strictly stationary $\mathrm{r}$ vector valued time series, we suppose know that the data $\mathrm{Xa}(\mathrm{t}),(\mathrm{t}=1,2, \ldots \mathrm{T})$ Which is the trade volume weakly of the Orascom Construction Industries since 1/1/2003 till 31/12/2008, where all observations are available of the series is available with some missing observations. $\mathrm{H}=1, \mathrm{Y}(\mathrm{t})=\mathrm{X}(\mathrm{t})$, which is the classic case and then suppose that there is some missing observations in randomly way, i.e., $\mathrm{H} \neq 1$, to compare two cases shown in Fig. 6 . The weakly trade volume data, $X(t), 2003-2008$ and The auto correlation function of the weakly trade volume data are shown in Fig. 1 and Fig. 2. The auto correlation and the partial auto correlation functions of the weakly trade volume data before smoothing are discussed and shown in Fig. 3.

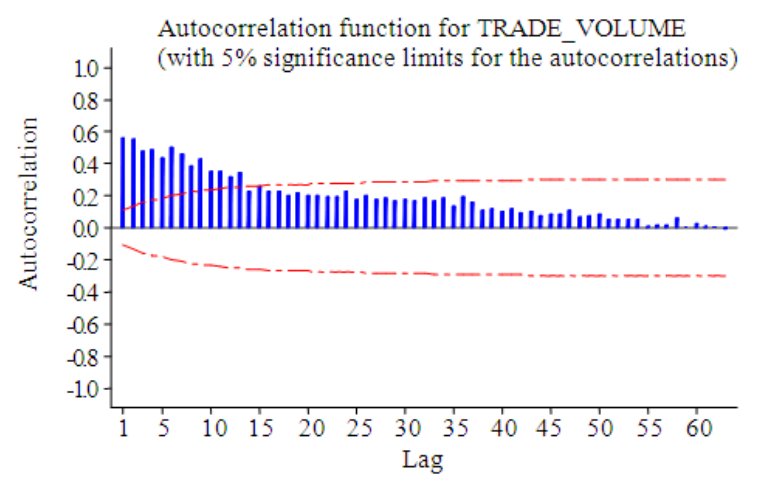

Fig. 2: The auto correlation function of the weakly trade volume data

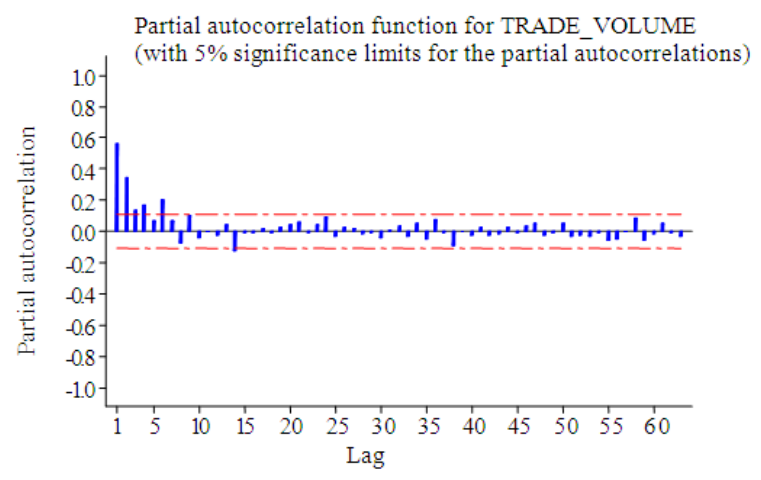

Fig. 3: The partial auto correlation function of the weakly trade volume data 


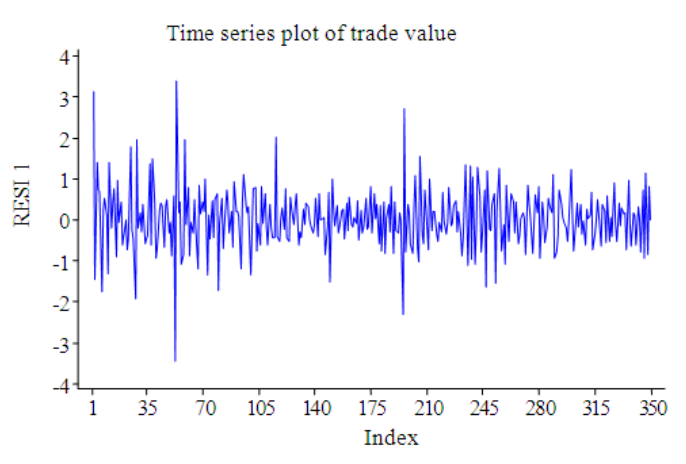

Fig. 4: The weakly trade volume data after adjustment $\mathrm{X}(\mathrm{t})$

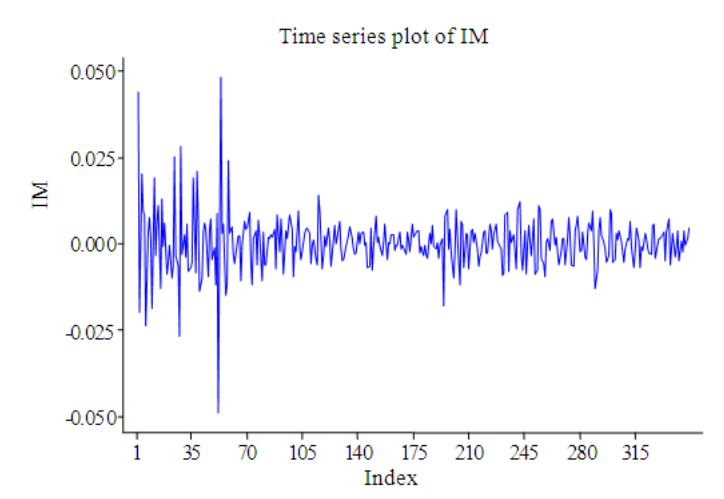

Fig. 5: The $f_{x}^{(\mathrm{T})}(\lambda), \quad f_{x}^{(\mathrm{T})}(\lambda)$ Imaginary part and imaginary part of $f_{x}^{(T)}(\lambda)$. where $p=0.99$

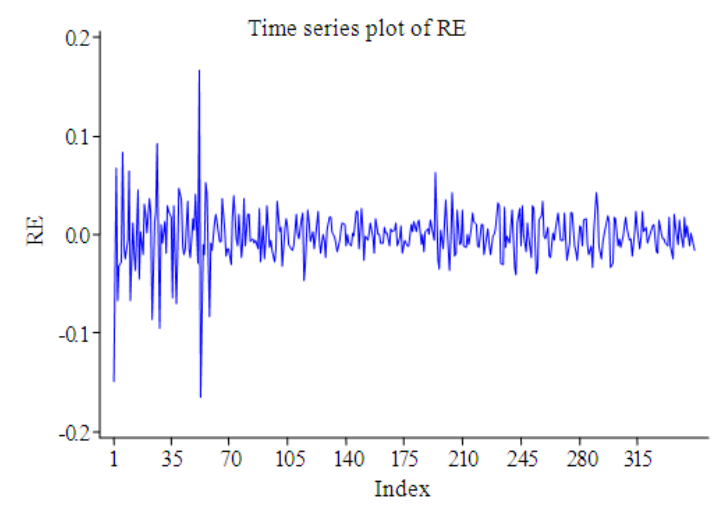

Fig. 6: The $f_{x}^{(\mathrm{T})}(\lambda), f_{x}^{(\mathrm{T})}(\lambda)$ real part and real part of $f_{x}^{(T)}(\lambda)$. where $p=0.99$

Figure 4 shows The weakly trade volume data after adjustment $\mathrm{X}(\mathrm{t}) \cdot \nabla_{1}$ is used to smooth data before calculations. The weakly trade volume data after adjustment $X(t)$ is mentioned. Figure 5 and 6 shows the imaginary part and real part of $f_{x}^{(T)}(\lambda)$. where $p=0.99$.
Table 1: box -pierce (ljung-box) chi-square statistic

\begin{tabular}{lllrrl}
\hline Type & Coef & SE & Coef & \multicolumn{1}{c}{ T } & P \\
\hline MA & 1 & 0.6670 & 10.0522 & 12.78 & 0.000 \\
MA & 2 & 0.1167 & 0.0524 & 2.23 & 0.027
\end{tabular}

Table 2: Modified box-pierce (ljung-box) chi-square statistic

\begin{tabular}{lrrrr}
\hline Lag & \multicolumn{1}{c}{12} & 24 & 36 & \multicolumn{1}{c}{48} \\
\hline Chi-square & 7.100 & 12.300 & 26.300 & 30.900 \\
DF & 10.000 & 22.000 & 34.000 & 46.000 \\
p-value & 0.721 & 0.951 & 0.823 & 0.957 \\
\hline
\end{tabular}

Table 1 shows the box-pierce (ljung-box) chi-square statistic and Table 2 shows Modified box-pierce (ljungbox) chi-square statistic.

\section{Final estimates of parameters:}

Differencing: 1 regular difference

Number of observations: Original series 350 , after differencing 349

Residuals: SS $=132.957$ (back forecasts excluded)

$\mathrm{MS}=0.383 \mathrm{DF}=347$

\section{MATERIALS AND METHODS}

We used SPSS and matlab, the software programming to solve our numerical example.

\section{RESULTS AND DISCUSSION}

The study of time series with missed observations and with the modified periodogram had the same results of the study of the classical time series.

\section{CONCLUSION}

Modified periodogram with expanded finite Fourier transformation for time series with missed observation has improved the results of the classic time series.

\section{REFERENCES}

Brillinger, D.R., 1969, Asymptotic properties of spectral estimates of second order. Biometrica, 56: 375-390. http://www.jstor.org/stable/2334430

Dahlhaus, R., 1985. Asymptotic normaity of spectral estimates. J. Multivar. Anal., 16: 412-431. http://ideas.repec.org/a/eee/jmvana/v16y1985i3p41 2-431.html

Ghazal, M.A. and E.A. Farag, 2000. Some properties of the continuous expanded finite Fourier transform. J. Stat., 3: 377-384. 
Ghazal, M.A., 2001. Statistical analysis of broanded periodogram for continuous time stationary process. J. Applied Math. Comput., 124: 343-349. DOI: 10.1016/S0096-3003(00)00099-0

Ghazal, M.A., 2005. Statistical properties of time series with missing observations. Int. J. Applied Math., 18: 143-155.
Ghazal, M.A. and A. Elhassanein, 2006. Periodogram analysis with missing observations. J. Applied Math. Comput., 22: 209-222. DOI: 10.1007/BF02896472 